\title{
ESTUDO DO EQUILÍBRIO DE FASES EM SISTEMAS AQUOSOS BIFÁSICOS COMPOSTOS POR LÍQUIDO IÔNICO, SAL E ÁGUA
}

\author{
M. C. PIGNATA ${ }^{1}$, R. C. F. BONOMO ${ }^{1}$ e R. C. S. SOUSA ${ }^{2}$ \\ ${ }^{1}$ Universidade Federal de Viçosa, Departamento de Química e Engenharia Química \\ ${ }^{2}$ Universidade Estadual do Sudoeste da Bahia, Departamento de Tecnologia Rural e Animal \\ E-mail para contato: rita.sousa@ufv.br
}

\begin{abstract}
RESUMO - Neste trabalho foram determinados diagramas de equilíbrio para sistemas aquosos bifásicos compostos por líquido iônico (LI), fosfato de potássio e água. Todos os diagramas foram determinados nas temperaturas de $20,30,40$ e $50{ }^{\circ} \mathrm{C}$ e em pH 7,5, 8,0 e 8,5. Constatou-se que não houve efeito da temperatura sobre a formação da região bifásica para os sistemas estudados. Quanto ao efeito do $\mathrm{pH}$, observou-se que o aumento do $\mathrm{pH}$ ocasionou um aumento da região bifásica em todas as temperaturas estudadas.
\end{abstract}

\section{INTRODUÇÃO}

Um método eficaz e economicamente viável para a separação e purificação de biomoléculas é a sua partição através de Sistemas Aquosos Bifásicos (SAB). SAB ocorrem da mistura de dois solutos mútuos e incompatíveis em água a partir da qual duas fases aquosas são formadas com diferentes composições. Solutos se distribuem entre as duas fases, dependendo da sua afinidade relativa por cada uma das fases individuais. Esta técnica é amplamente utilizada na purificação de biomoléculas e suas vantagens incluem seletividade favorável, baixo custo e adaptabilidade para o processamento contínuo da amostra e retenção da atividade biológica (Selber et al, 2001). Os sistemas mais estudados são os formados por polietileno glicol-dextrana (PEG-dextrana) e PEGSal. No entanto, a maior parte dos polímeros dos SAB possuem alta viscosidade (Mei et al, 1995; Perumalsamy et al, 2007) e normalmente formam soluções aquosas opacas, que poderiam interferir com a análise quantitativa e qualitativa dos compostos extraídos.

Durante os últimos anos, os SAB baseados em Líquidos Iônicos (LIs) têm sido aplicados em processos de biopurificação e bio-extração, pois LI são substancialmente menos viscosos do que os típicos SAB à base de polímeros (Cláudio et al., 2010; Louros et al., 2010).

Para otimizar o emprego dos SAB na partição de biocompostos é necessário o estudo do comportamento destes sistemas em diferentes composições e em variadas condições de temperatura e $\mathrm{pH}$. Assim, o objetivo deste trabalho foi determinar dados de equilíbrio dos sistemas compostos por cloreto de 1-etil-3-metilimidazólio + fosfato de potássio + água, nos valores de $\mathrm{pH}$ $7,5,8,0$ e 8,5 e nas temperaturas de $(20,30,40 \text { e } 50)^{\circ} \mathrm{C}$.

\subsection{Sistemas Aquosos Bifásicos (SAB) baseados em líquidos iônicos}

SAB comuns, geralmente, são formados por polietileno glicol (PEG) porque este, facilmente, forma um sistema bifásico com sais inorgânicos e polímeros neutros. A presença de 
um sal inorgânico em concentrações críticas em um sistema único polímero-água pode levar à formação de duas fases aquosas distintas, onde normalmente a fase inferior é rica em sal inorgânico, enquanto a fase superior é rica em polímero.

Polímeros podem ser substituídos diretamente usando LIs hidrofóbicos como uma fase líquida imiscível em meios aquosos (Tomé et al., 2010) ou pelo uso de SAB incorporando LIs hidrofílicos (Gutowski et al., 2003; Louros et al., 2010). A segunda abordagem é mais usada porque os SAB contendo LIs hidrofílicos mostraram-se mais eficazes na extração de biomoléculas (Tomé et al., 2010; Ventura et al., 2009). Esses novos SAB também têm muitas vantagens oferecidas pelo uso de LIs, tais como baixa viscosidade, pouca formação de emulsão, não há necessidade do uso de solvente orgânico volátil, separação de fases rápida e extração de alta eficiência.

Líquidos Iônicos: Os líquidos iônicos (LIs), são eletrólitos formados pela combinação de um cátion assimétrico volumoso com um ânion fracamente coordenante, pertencentes a uma classe de solventes com pontos de fusão abaixo de $100{ }^{\circ} \mathrm{C}$ e reúnem um conjunto de características interessantes, tais como, líquidos sob uma ampla faixa de temperatura, negligenciável pressão de vapor, desprezível inflamabilidade, baixa toxicidade (solvente verde), boa estabilidade química e térmica, habilidade catalítica e alta condutividade iônica (Wei e Ivaska, 2008).

Diagrama de fases: Entende-se como diagrama de equilíbrio uma representação gráfica utilizada para expressar as concentrações de um sistema de fases. Os dados de equilíbrio são representados a temperatura e pressão constantes. Para a utilização de SAB é necessário o conhecimento do comportamento das fases nos sistemas. Para isto são obtidos os diagramas de fases para os componentes, nos quais as composições dos constituintes para a separação das fases são determinadas (Kabiri-Badr e Cabezas Jr., 1996).

\section{MATERIAL E MÉTODOS}

O trabalho experimental foi realizado no Laboratório de Engenharia de Processos da Universidade Estadual do Sudoeste da Bahia, campus de Itapetinga. Os reagentes utilizados na condução deste trabalho foram: cloreto de 1-etil-3-metilimidazólio $\left(\left[\mathrm{C}_{2} \mathrm{~min}\right] \mathrm{Cl}\right)$ da Sigma-Aldrich (USA) com grau de pureza $\geq 95 \%$ e fosfato de potássio monobásico $\left(\mathrm{KH}_{2} \mathrm{PO}_{4}\right)$ e bibásico $\left(\mathrm{K}_{2} \mathrm{HPO}_{4}\right)$, adquiridos da Vetec (Brasil) e Cinética (Brasil), respectivamente. Nos experimentos foram empregados água destilada e todos os reagentes usados foram de grau analítico.

Soluções estoques dos reagentes foram preparadas, com concentrações iguais a $60 \% \mathrm{~m} / \mathrm{m}$ para o LI e $40 \% \mathrm{~m} / \mathrm{m}$ para o sal (fosfato) nos valores de $\mathrm{pH} 7,5,8,0$ e 8,5, pela mistura do fosfato de potássio monobásico e bibásico, conferidos em pHmetro e ajustados quando necessários.

\subsection{Determinação dos Dados de Equilíbrio de Fases}

Diagramas de equilíbrio para os $\mathrm{SAB} \mathrm{LI}\left\{\left[\mathrm{C}_{2} \mathrm{~min}\right] \mathrm{Cl}\right\}$-fosfato de potássio foram obtidos experimentalmente nas temperaturas de $(20,30,40 \text { e } 50)^{\circ} \mathrm{C}$ e nos valores de $\mathrm{pH} 7,5,8,0$ e 8,5. As curvas binodais foram determinadas pelo método turbidimétrico (Albertsson, 1986). A equação empírica (Equação 1) sugerida por $\mathrm{Hu}$ et al. (2003) foi ajustada aos dados das curvas binodais experimentais: 


$$
X_{L I}=\exp \left(a+b\left(X_{S A L}\right)^{0,5}+c X_{S A L}+d\left(X_{S A L}\right)^{2}\right)
$$

em que $\mathrm{X}_{\mathrm{LI}}$ e $\mathrm{X}_{\mathrm{SAL}}$ são as composições em fração mássica do LI e sal, respectivamente, e a, b, c e d são parâmetros de ajuste da equação.

As linhas de amarração (LA) foram obtidas utilizando o método gravimétrico. Para isso, foi selecionado primeiro um ponto dentro da região bifásica, através da qual a LA vai passar. Foram utilizados três pontos globais para cada sistema LI-sal. Para cada condição de $\mathrm{pH}$ e temperatura, foi escolhido um ponto que deu origem a LA, com composições conhecidas. O sistema foi preparado em tubos de centrífuga graduados de fundo cônico, a partir da massa das soluções estoques de LI, sal e água resultando em uma massa total do sistema de $40 \mathrm{~g}$.

Quantificação dos Componentes do Sistema: Depois das fases terem sido separadas, elas foram pesadas. A concentração de cada componente nas fases coletadas foi estimada pela aplicação da regra da alavanca, na relação entre a composição em massa da fase superior e a composição total do sistema (Merchuck et al., 1998). Para determinação dos componentes da LA o seguinte sistema de quatro equações (Equações 2-5) e quatro valores desconhecidos $\left(X_{L I}{ }^{s}, X_{L I}{ }^{i}\right.$, $X_{S A L}{ }^{s}, X_{S A L}{ }^{i}$ ) foi resolvido (Hu et al., 2003) através do programa Solver.

$$
\begin{aligned}
& X_{L I} s=\exp \left(a+b\left(X_{S A L} s\right)^{0,5}+c X_{S A L} s+d\left(X_{S A L} s\right)^{2}\right) \\
& X_{L I^{i}}=\exp \left(a+b\left(X_{S A L}\right)^{0,5}+c X_{S A L^{i}}+d\left(X_{S A L}\right)^{2}\right) \\
& \frac{X_{L I}{ }^{s-X_{L I} M}}{X_{L I^{M}}-X_{L I^{i}}}=\frac{m_{i}}{m_{S}} \\
& \frac{X_{S A L^{M}}-X_{S A L^{s}}}{X_{S A L^{i}}-X_{S A L^{M}}}=\frac{m_{i}}{m_{S}}
\end{aligned}
$$

\section{RESULTADOS E DISCUSSÃO}

\subsection{Efeito da temperatura e do pH nos diagramas de equilíbrio}

Foram obtidos diagramas de equilíbrio de fases para os SAB estudados em função da temperatura e dos valores de $\mathrm{pH}$. Os diagramas de fases para os sistemas LI-Fosfato são apresentados nas Figuras 1, 2 e 3. Os dados obtidos experimentalmente por turbidimetria na obtenção das curvas binodais dos sistemas foram correlacionados pela equação não linear (Equação 1) proposta por Hu et al. (2003). 




Figura 1 - Diagrama de fase $\left[\mathrm{C}_{2} \mathrm{~min}\right] \mathrm{Cl}$-Fosfato de potássio em $\mathrm{pH}$ 7,5 para diferentes temperaturas.

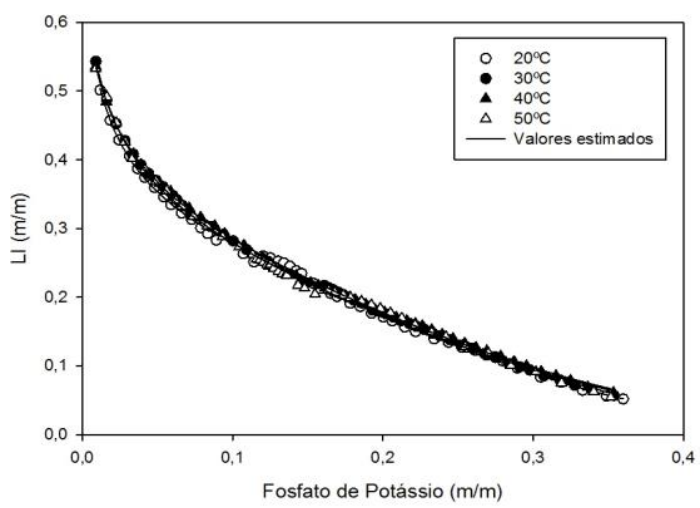

Figura 2 - Diagrama de fase $\left[\mathrm{C}_{2} \mathrm{~min}\right] \mathrm{Cl}$-Fosfato de potássio em $\mathrm{pH}$ 8,0 para diferentes temperaturas.



Figura 3 - Diagrama de fase $\left[\mathrm{C}_{2} \mathrm{~min}\right] \mathrm{Cl}-$ Fosfato de potássio em $\mathrm{pH}$ 8,5 para diferentes temperaturas.

Observando-se as Figuras 1 a 3, pode-se notar que os sistemas não sofreram variação da região bifásica com o aumento da temperatura. Isto indica que há uma variação insignificante da capacidade calorífica das fases e, consequentemente, baixa variação de entalpia associada ao processo de separação. Os valores obtidos para os parâmetros a, b, c e d da Equação 1 com os seus correspondentes desvios padrão e os coeficientes de determinação $\left(\mathrm{R}^{2}\right)$ das equações ajustadas 
para os sistemas investigados estão apresentados na Tabela 1.

Tabela 1 - Parâmetros $a, b, c$, e $d$ e o coeficiente de determinação $\left(\mathrm{R}^{2}\right)$ obtidos para o sistemas LI-

Fosfato de potássio pela equação de Hu et al. (2003)

\begin{tabular}{|c|c|c|c|c|c|}
\hline $\mathrm{pH}$ & $a$ & $b$ & $c$ & $d$ & $\mathrm{R}^{2}$ \\
\hline \multicolumn{6}{|c|}{$20^{\circ} \mathrm{C}$} \\
\hline 7,5 & $-0,5108 \pm 0,0278$ & $-1,5229 \pm 0,2868$ & $-1,4142 \pm 0,7331$ & $-4,7410 \pm 1,1036$ & 0,9986 \\
\hline 8,0 & $-0,1964 \pm 0,0298$ & $-5,3142 \pm 0,2975$ & $7,8084 \pm 0,7541$ & $-18,3799 \pm 1,1469$ & 0,9984 \\
\hline 8,5 & $-0,2572 \pm 0,0197$ & $-5,0839 \pm 0,2014$ & $7,7165 \pm 0,5130$ & $-19,5849 \pm 0,7717$ & 0,9994 \\
\hline \multicolumn{6}{|c|}{$30^{\circ} \mathrm{C}$} \\
\hline 7,5 & $-0,2677 \pm 0,0132$ & $-3,8454 \pm 0,1411$ & $3,5835 \pm 0,3807$ & $-9,4653 \pm 0,6220$ & 0,9995 \\
\hline 8,0 & $-0,2079 \pm 0,0119$ & $-4,7822 \pm 0,1238$ & $6,1876 \pm 0,3235$ & $-15,6435 \pm 0,5172$ & 0,9996 \\
\hline 8,5 & $-0,2149 \pm 0,0108$ & $-4,2535 \pm 0,1130$ & $4,2910 \pm 0,2988$ & $-12,0931 \pm 0,4797$ & 0,9997 \\
\hline \multicolumn{6}{|c|}{$40^{\circ} \mathrm{C}$} \\
\hline 7,5 & $-0,2147 \pm 0,0143$ & $-3,7667 \pm 0,2740$ & $3,1060 \pm 0,6713$ & $-8,7924 \pm 0,9704$ & 0,9991 \\
\hline 8,0 & $-0,2747 \pm 0,0143$ & $-4,0833 \pm 0,1480$ & $4,3996 \pm 0,3845$ & $-12,5254 \pm 0,6061$ & 0,9994 \\
\hline 8,5 & $-0,2827 \pm 0,0164$ & $-3,4917 \pm 0,1740$ & $4,5091 \pm 0,4532$ & $-14,1878 \pm 0,7086$ & 0,9993 \\
\hline \multicolumn{6}{|c|}{$50^{\circ} \mathrm{C}$} \\
\hline 7,5 & $-0,4657 \pm 0,0155$ & $-1,7077 \pm 0,1695$ & $-0,8957 \pm 0,4573$ & $-6,5975 \pm 0,7398$ & 0,9994 \\
\hline 8,0 & $-0,2407 \pm 0,0314$ & $-4,4036 \pm 0,3308$ & $4,8628 \pm 0,8670$ & $-12,9329 \pm 1,3893$ & 0,9971 \\
\hline 8,5 & $-0,3653 \pm 0,0373$ & $-3,1762 \pm 0,3759$ & $1,7832 \pm 0,9499$ & $-7,9150 \pm 1,4307$ & 0,9980 \\
\hline
\end{tabular}

\subsection{Estudo do comportamento das linhas de amarração}

A partir da caracterização dos diagramas de fases foram obtidas as linhas de amarração pelo método gravimétrico e pela aplicação da regra da alavanca. As composições de equilíbrio para os sistemas estudados nas diferentes condições de temperatura e pH estão apresentadas na Tabela 2, em que os dados referentes aos componentes das fases estão expressos em termos de porcentagem mássica. Três linhas de amarração foram determinadas para cada sistema estudado. O comprimento da linha de amarração (CLA) representa a diferença entre a concentração de sal inorgânico e de LI nas fases superior e inferior, quanto maior o CLA, maior é a concentração de LI na fase superior e a concentração do sal na fase inferior.

De acordo com os resultados apresentados na Tabela 2, pode-se observar que a concentração de LI na fase inferior para a maioria dos sistemas, é muita baixa, e em alguns casos, o LI é quase totalmente excluído dessa fase, enquanto que o comportamento contrário é observado na fase superior. A exclusão é mais pronunciada com a diminuição do valor de $\mathrm{pH}$, nas temperaturas de $20{ }^{\circ} \mathrm{C}$ e $40{ }^{\circ} \mathrm{C}$ e na temperatura de $50{ }^{\circ} \mathrm{C}$ ao reduzir o $\mathrm{pH}$ de 7,5 pra 8,0 . Este comportamento de exclusão é comum à maioria dos SAB formados por LI-Sal, sendo que a fase superior é composta majoritariamente por LI e água, e a fase inferior é composta principalmente por sal e água. A composição total do sistema não tem efeito sobre a inclinação das linhas de amarrações, o que implica que as LAs são paralelas entre si. 
Os valores do comprimento da linha de amarração (CLA) para cada sistema estudado também estão na Tabela 2.

Tabela 2 - Dados das linhas de amarrações e porcentagem mássica $(\% \mathrm{~m} / \mathrm{m})$ dos sistemas formados por LI $\left\{\left[\mathrm{C}_{2} \mathrm{~min}\right] \mathrm{Cl}\right\}\left(\mathrm{W}_{1}\right)+$ Fosfato de Potássio $\left(\mathrm{W}_{2}\right)+$ Água, em diferentes valores de $\mathrm{pH} \mathrm{e}$ temperatura

\begin{tabular}{|c|c|c|c|c|c|c|c|c|}
\hline \multirow[b]{2}{*}{$\mathrm{pH}$} & \multirow[b]{2}{*}{ LA } & \multicolumn{2}{|c|}{ Composição Global } & \multicolumn{2}{|c|}{ Fase Superior } & \multicolumn{2}{|c|}{ Fase Inferior } & \multirow[b]{2}{*}{ CLA } \\
\hline & & $\mathrm{W}_{1}$ & $\mathrm{~W}_{2}$ & $\mathrm{~W}_{1}$ & $\mathrm{~W}_{2}$ & $\mathrm{~W}_{1}$ & $\mathrm{~W}_{2}$ & \\
\hline & \multicolumn{8}{|c|}{$20^{\circ} \mathrm{C}$} \\
\hline \multirow{4}{*}{7,5} & 1 & 22,00 & 21,00 & 31,58 & 9,38 & 5,87 & 40,54 & 40,40 \\
\hline & 2 & 23,02 & 21,98 & 35,63 & 6,91 & 4,71 & 43,88 & 48,20 \\
\hline & 3 & 24,00 & 23,00 & 38,76 & 5,25 & 3,70 & 47,41 & 54,83 \\
\hline & 1 & 21,99 & 18,01 & 29,75 & 8,44 & 2,83 & 41,67 & 42,77 \\
\hline \multirow[t]{3}{*}{8,0} & 2 & 23,01 & 19,00 & 34,02 & 5,67 & 2,04 & 44,36 & 50,20 \\
\hline & 3 & 24,04 & 19,97 & 37,46 & 4,08 & 1,52 & 46,65 & 55,71 \\
\hline & 1 & 20,00 & 20,00 & 27,41 & 10,12 & 1,69 & 44,41 & 42,86 \\
\hline \multirow[t]{4}{*}{8,5} & 2 & 21,01 & 20,99 & 31,50 & 6,94 & 1,06 & 47,73 & 50,90 \\
\hline & 3 & 21,98 & 22,03 & 34,66 & 5,05 & 0,69 & 50,53 & 56,77 \\
\hline & \multicolumn{8}{|c|}{$30^{\circ} \mathrm{C}$} \\
\hline & 1 & 19,99 & 22,01 & 27,43 & 11,72 & 5,21 & 42,45 & 37,92 \\
\hline \multirow[t]{3}{*}{7,5} & 2 & 21,00 & 23,00 & 30,95 & 8,95 & 3,42 & 47,82 & 47,63 \\
\hline & 3 & 22,01 & 23,99 & 34,07 & 6,93 & 2,47 & 51,64 & 54,75 \\
\hline & 1 & 20,04 & 20,98 & 28,29 & 10,11 & 2,48 & 44,11 & 42,69 \\
\hline \multirow[t]{3}{*}{8,0} & 2 & 20,99 & 22,00 & 31,85 & 7,56 & 1,60 & 47,83 & 50,37 \\
\hline & 3 & 22,00 & 23,00 & 35,10 & 5,68 & 1,13 & 50,58 & 56,30 \\
\hline & 1 & 19,99 & 21,00 & 28,67 & 9,95 & 3,80 & 41,64 & 40,28 \\
\hline \multirow[t]{4}{*}{8,5} & 2 & 21,01 & 21,99 & 32,35 & 7,55 & 2,68 & 45,34 & 48,05 \\
\hline & 3 & 22,00 & 22,99 & 35,92 & 5,68 & 2,10 & 47,75 & 53,98 \\
\hline & \multicolumn{8}{|c|}{$40^{\circ} \mathrm{C}$} \\
\hline & 1 & 22,02 & 21,98 & 28,17 & 11,86 & 1,87 & 55,22 & 50,71 \\
\hline \multirow[t]{2}{*}{7,5} & 2 & 23,00 & 22,99 & 30,69 & 9,96 & 1,19 & 60,01 & 58,10 \\
\hline & 3 & 24,02 & 23,99 & 33,07 & 8,38 & 0,79 & 64,04 & 64,34 \\
\hline \multirow[t]{4}{*}{8,0} & 1 & 21,00 & 20,00 & 25,68 & 12,32 & 1,24 & 52,48 & 47,01 \\
\hline & 2 & 21,99 & 21,00 & 28,34 & 10,14 & 0,68 & 57,47 & 54,82 \\
\hline & 3 & 22,90 & 22,07 & 30,59 & 8,51 & 0,38 & 61,84 & 61,29 \\
\hline & 1 & 20,97 & 21,03 & 26,61 & 12,27 & 0,98 & 52,07 & 47,34 \\
\hline \multirow[t]{4}{*}{8,5} & 2 & 21,99 & 22,00 & 29,35 & 10,12 & 0,53 & 56,62 & 54,71 \\
\hline & 3 & 23,00 & 23,00 & 32,25 & 8,09 & 0,35 & 59,50 & 60,50 \\
\hline & \multicolumn{8}{|c|}{$50^{\circ} \mathrm{C}$} \\
\hline & 1 & 21,99 & 22,00 & 28,23 & 12,10 & 1,54 & 54,49 & 50,09 \\
\hline \multirow[t]{3}{*}{7,5} & 2 & 23,01 & 22,99 & 30,75 & 10,37 & 1,02 & 58,84 & 56,86 \\
\hline & 3 & 24,01 & 23,99 & 33,10 & 8,87 & 0,68 & 62,80 & 62,92 \\
\hline & 1 & 22,00 & 19,99 & 27,68 & 10,17 & 0,69 & 56,86 & 53,93 \\
\hline \multirow[t]{3}{*}{8,0} & 2 & 22,99 & 21,00 & 30,44 & 8,19 & 0,47 & 59,73 & 59,62 \\
\hline & 3 & 24,00 & 22,00 & 33,10 & 6,57 & 0,34 & 62,08 & 64,46 \\
\hline & 1 & 22,00 & 19,00 & 27,46 & 10,45 & 2,63 & 49,30 & 46,11 \\
\hline \multirow[t]{2}{*}{8,5} & 2 & 23,00 & 19,99 & 30,26 & 8,47 & 1,81 & 53,61 & 53,36 \\
\hline & 3 & 24,00 & 21,00 & 33,21 & 6,68 & 1,46 & 56,03 & 58,68 \\
\hline
\end{tabular}


Segundo Da Silva e Loh (2006), o CLA está associado à seletividade do sistema. Quanto menor o CLA, associada a uma dada composição global do sistema, mais próximo da unidade será o valor do coeficiente de partição da biomolécula de interesse, tornando-se menos seletiva a extração. No entanto, com o aumento do CLA, a biomolécula de interesse tende a migrar mais para uma das fases, isto é, o valor do coeficiente de partição torna-se cada vez mais distante de 1, para mais ou para menos, aumentando a eficiência de extração. Neste estudo, pode-se observar que à medida que aumenta o valor de $\mathrm{pH}$ o CLA também aumenta, com o aumento do $\mathrm{pH}$ a razão $\mathrm{H}_{2} \mathrm{PO}_{4}{ }^{-} / \mathrm{HPO}_{4}^{-2}$ diminui, aumentando o efeito salting-out do LI, e consequentemente, aumentando a diferença de composição entre fases.

A partir dos dados apresentados na Tabela 2, observa-se que a temperatura influencia no comprimento e na inclinação das linhas de amarração dos sistemas. Esse comportamento é observado em todos os valores de $\mathrm{pH}$ estudados. Os valores absolutos da inclinação das linhas de amarração diminuem ligeiramente com o aumento da temperatura. Pode-se dizer que a redução da temperatura aumenta a afinidade do fosfato de potássio pela água induzindo uma migração de água para longe dos íons de LI reduzindo a sua hidratação e solubilidade em água do LI (Freire et al., 2010), ou seja, a água da fase rica em LI foi expulsa para a fase rica em sal pela diminuição da temperatura, diminuindo a concentração de sal na fase inferior e, como consequência, um aumento da ILA. No entanto, ao comparar as temperaturas de $40{ }^{\circ} \mathrm{C}$ e $50{ }^{\circ} \mathrm{C}$, observa-se que tanto o CLA quanto a ILA foram bem próximos para todos os valores de $\mathrm{pH}$. Ao estudar a interferência do $\mathrm{pH}$ na composição das fases, nota-se que a variação de $\mathrm{pH}$ não interferiu na inclinação da linha de amarração (Figura 3).

\section{CONCLUSÃO}

Foram determinados dados de equilíbrio para sistemas aquosos formados por LI $\left\{\left[\mathrm{C}_{2} \min \right] \mathrm{Cl}\right\}+$ Fosfato de Potássio + Água, nas temperaturas de $20,30,40,50{ }^{\circ} \mathrm{C}$ nos valores de $\mathrm{pH} 7,5,8,0$ e 8,5. Constatou-se que o efeito da temperatura sobre os sistemas não apresentou variações na região bifásica. Houve um aumento na inclinação da linha de amarração à medida que a temperatura diminuía, podendo dizer que ocorreu transferência das moléculas de água da fase superior para a fase inferior. Pôde-se também constatar que o aumento do $\mathrm{pH}$ ocasionou um aumento da região bifásica em todas as temperaturas de estudo.

\section{AGRADECIMENTOS}

Os autores agradecem à Fapemig e ao CNPq pelo apoio financeiro.

\section{REFERÊNCIAS}

ALBERTSSON, P.A. Aqueous Polymer-phase Systems Partition of Cell Particles and Macromolecules, 3nd edition, Wiley, New York, 1986.

CLÁUDIO, A.F.M.; FREIRE, M.G.; FREIRE, C.S.R.; SILVESTRE, A.J.D.; COUTINHO, J.A.P. Extraction of Vanillin using Ionic-Liquid-Based Aqueous Two-Phase Systems. Separation and Purification Technology, v.75, p.39-47, 2010.

FREIRE, M.G., NEVES, C.; SILVA, A.M.S.; SANTOS, L. M. N. B. F.; MARRUCHO, I.M.; REBELO, L.P.N.; SHAH,J.K.; MAGINN, E.J.; COUTINHO, J.A.P. H-1 NMR and 
Molecular Dynamics Evidence for an Unexpected Interaction on the Origin of SaltingIn/Salting-Out Phenomena. Journal of Physical Chemistry B, v.114, n.5, p. 2004-2014, 2010 .

GUTOWSKI, K.E.; BROKER, G.A.; WILLAUER, H.D.; HUDDLESTON, J.G.; SWATLOSKI, R.P.; HOLBREY, J.D.; ROGERS, R.D. Controlling the aqueous miscibility of ionic liquids: Aqueous biphasic systems of water-miscible ionic liquids and water-structuring salts for recycle, metathesis, and separations. Journal of the American Chemical Society, v.125, n.22, p.6632-6633, 2003.

KABIRI-BADR, M. and CABEZAS JR., H. A thermodynamic model for the phase behavior of salt-polymer aqueous two-phase systems. Fluid Phase Equilibria, v.115, n.2, p. 39-58, 1996.

LOUROS, C.L.S.; CLÁUDIO, A.F.M; NEVES, C.M.S.S.; FREIRE, M.G.; MARRUCHO, I.M.; PAULY, J.; and COUTINHO, J.A.P. Extraction of Biomolecules Using PhosphoniumBased Ionic Liquids $+\mathrm{K}_{3} \mathrm{PO}_{4}$ Aqueous Biphasic Systems. International Journal of Molecular Sciences, v.11, n.4, p.1777-1791, 2010.

MERCHUK, J. C.; ANDREWS, B. A.; ASENJO, J. A. Aqueous two-phase systems for protein separation. Studies on phase inversion. Journal Chromatography B, v.711, p.285-293, 1998.

MEI, L.H.; LIN, D.Q.; ZHU, Z.Q.; HAN, Z.X. Densities and viscosities of polyethylene glycol + salt + water systems at $20^{\circ} \mathrm{C}$. Journal Chemical Engineering Data, v.40, p.1168-1171, 1995.

NEVES, C.; VENTURA, S.P.M.; FREIRE, M.G.; MARRUCHO, I.M.; COUTINHO, J.A.P. Evaluation of Cation Influence on the Formation and Extraction Capability of Ionic-LiquidBased Aqueous Biphasic Systems. Journal of Physical Chemistry B, v.113, n.15, p.51945199, 2009.

PERUMALSAMY, M.; BATHMALAKSHMI, A.; MURUGESAN, T. Experiment and Correlation of Liquid-Liquid Equilibria of an Aqueous Salt Polymer System Containing PEG6000 + Sodium Citrate. Journal of Chemical Engineering Data, v.52, p.1186-1188, 2007.

SELBER, K.; COLLEN, A.; HYYTIA, T.; PENTTILA, M.; TJERNELD, F.; KULA, MR. Parameters influencing protein extraction for whole broths in detergent based aqueous twophase systems. Bioseparation, v.10, p.229-236, 2001.

TOMÉ, L.I.N.; CATAMBAS, V.R.; TELES, A.R.R.; FREIRE, M.G.; MARRUCHO, I.M.; COUTINHO, J.A.P. Tryptophan extraction using hydrophobic ionic liquids. Separation and Purification Technology, v.72, n.2, p.167-173, 2010.

VENTURA, S. P. M.; SOUSA, S. G.; FREIRE, M. G.; SERAFIM, L. S.; LIMA, A. S. Design of ionic liquids for lípase purification. Journal of Chromatography B, v.879, p.2679-2687, 2011.

WEI, D.; IVASKA, A. Applications of ionic liquids in electrochemical sensors. Analytica Chimica Acta, v.607, n.2, p.126-135, 2008. 